\title{
La innovación, el desarrollo y la propiedad intelectual en la universidad
}

\author{
Alberto Urbina*
}

\section{RESUMEN}

La universidad a lo largo de su historia ha sufrido cambios científico-académicos, administrativos y políticos; obedeciendo a la influencia de su entorno nacional e internacional, según el modelo económico predominante. Ha seguido arrastrando un rezago en función de la dinámica del desarrollo socio-económico y, lo que es peor, mientras el sector productivo se innova permanentemente, la universidad se aferra a la tradición profesionalizante, incluso desconociendo la demanda real; formando profesionales en carreras tradicionales con conocimientos en franca obsolescencia y limitadas posibilidades de competir en el mercado, salvando desde luego las escasas excepciones.

Las universidades que se resisten al cambio y a la mejora continua, están destinadas a la deslegitimización y la autolimitación en su propio desarrollo, y tendrán muy pocas posibilidades en su justificación social de existir como tales. El reto está planteado y es repensarse a sí mismas de conformidad a la denominada sociedad del conocimiento. Desde luego que esto implica asumir compromisos serios en diferentes ámbitos; empezando por conocer sus fortalezas y debilidades, superar las debilidades $y$, consecuentemente, asumir el papel fundamental de producir, transmitir y difundir el conocimiento, desarrollando con responsabilidad la cuarta misión, que se suma a las tradicionales de docencia, investigación y vinculación, definiéndola en términos de "uso, aplicación y explotación del conocimiento".

La universidad tiene la responsabilidad histórica de superar el viejo paradigma de simple transmisora de conocimientos, observadora pasiva de la realidad y descriptora de problemas sociales, económicos y ambientales. Debe desafiar su propia tradición; construyendo un liderazgo propositivo, definido por la innovación y el uso intensivo de las nuevas tecnologías, tanto en la función de educadora como en la transferencia de activos intangibles mediados por la propiedad intelectual y fortaleciendo el tejido productivo y social.

\footnotetext{
*Universidad Nacional Autónoma de Honduras, Dirección de Investigación Científica innovagroseire21@yahoo.com
} 
Palabras clave: Investigación, innovación, desarrollo, propiedad intelectual, currículo.

\begin{abstract}
Throughout history the university has undergone academical, scientifical, administrative and political changes due to the influence of its national and international environment, in accordance with the predominant economical model, however it has not been able to keep up with the socio economical development dynamics, and even worse, while the productive sector is permanently innovating, the university sticks to its professionalizing tradition, sometimes even ignoring the real social demands, forming professionals in traditional careers with obsolete knowledge and limited possibilities to compete in the market, with a very few exceptions.
\end{abstract}

Universities that resist change and permanent improvement are destined to be illegitimate and limited in their own growth. They will have very few social reasons to exist as such. The challenge is to rethink itself according to the knowledge society. This, of course, implies the assumption of serious commitments in different areas, beginning with an analysis of its strengths and weaknesses, overcoming those weaknesses and consequently assuming its fundamental role of producing, transmitting and publishing knowledge. The university has to responsibly perform its fourth mission that is added to the traditional activities of teaching, research and extension, defined in terms of "usage, application and exploitation of knowledge".

The university has the historical responsibility of surpassing the old paradigm of simply transmitting knowledge, of passively observing reality and describing social, economical and environmental problems. It must challenge its own tradition constructing a proactive leadership defined by innovation and the intensive usage of new technologies, both in the educative function as well as in the intangible assets mediated by intellectual property and strengthening the productive and social tissue.

Key words: Research, innovation, development, intellectual property, curriculum. 


\section{INTRODUCCIÓN}

Si la cadena de la innovación la eslabonan la universidad, el tejido productivo y social, los recursos de I+D, las actuaciones públicas y el entorno socio-económico; con enfoque de sistema, interrelacionados sus componentes e interactuando de manera interdependiente, es perentorio que la universidad proceda a reconstruir su tejido académico fortaleciendo las sinergias correspondientes al sistema de innovación. De acuerdo con Marco Romilio Estrada (2004), en su propuesta de Plan Estratégico 2005-2010 del Sistema Centroamericano de Relación UniversidadSociedad, la innovación "refleja un estado determinado de conocimiento, un entorno institucional particular, una disponibilidad de aptitudes para definir un problema y resolverlo, cuya aplicación sea útil y difundible. Los innovadores aprenden creando, mientras que los no innovadores aprenden utilizando..."

Ser universidad innovadora demanda orientar sus capacidades hacia la gestión del conocimiento por medio de la investigación científica, el desarrollo tecnológico, la transferencia y la propiedad intelectual; por lo que inevitablemente deben favorecerse cambios estructurales, presupuestarios, normativos y culturales, realizar buenas prácticas en su oferta curricular, de investigación, desarrollo tecnológico, aprendizajes permanentes de sus actores, construcción de la cultura de propiedad intelectual y de vínculos con el sector productivo y particularmente crear mecanismos de interfaz con la comunidad científica internacional y los cooperantes nacionales e internacionales. La Universidad debe comprender que si no promueve la investigación científica, las posibilidades de incursionar con acierto e incidir en el desarrollo académico, social y económico de manera significativa, serán escasas y menos aún podrá innovar sus procesos, servicios y/o productos.

\section{MISIÓN DE LAUNIVERSIDAD}

Existe un trípode epistemológico, sobre el cual descansa la misión histórica de la Universidad frente a la ciencia, la tecnología y la sociedad: a) Producir conocimiento mediante la investigación científica, b) Transferir el conocimiento a través de la educación y la tecnología y c) Difundir el conocimiento mediante publicaciones. Este proceso pone en perspectiva la oportunidad de explotación del conocimiento con su valor agregado y su correspondiente protección de los derechos cognitivos y tecnológicos.

La misión de la universidad debe leerse no desde la historia sino en la historia misma; estudiando lo que entendemos por realidad en ese entramado multidimensional, integrado por la multidisciplinariedad que exige estrategias de 
gestión y operabilidad en equipos de actores que interpreten los códigos sociales y económicos, en sus desafíos planteados como problemas y falencias ontológicas, para impulsar soluciones sostenibles. Lo anterior es coherente con la Declaración de la UNESCO en 1999, sobre "La ciencia y el uso del saber científico", ésta plantea: "El progreso del conocimiento mediante la investigación científica es una función esencial de todos los sistemas de educación superior, que tienen el deber de promover los estudios de Post-grado, la innovación y la interdisciplinariedad".

La Universidad no puede operar descontextualizada, ignorando que existen lazos naturales con su entorno: tejido productivo, instituciones públicas, entorno socioeconómico, ámbito de Investigación mas Desarrollo (I+D). Sin embargo, para crear y desarrollar las sinergias necesarias y engendrar confianza y liderazgo, necesita promover y estimular sus potencialidades internas y el uso de herramientas de generación, codificación, transferencia, implementación y propiedad intelectual.

El gran reto es reorganizar los procesos de generación, aprovechamiento del conocimiento y la cultura organizacional; lo que pasa por la formación y capacitación de profesionales, investigadores y gestores de activos intangibles, diseño y ejecución de políticas, estrategias de ciencia y tecnología, redefinición de normativas consecuentes con la sociedad del conocimiento, fortalecimiento de la infraestructura de I+D (laboratorios, observatorios, bibliotecas físicas y virtuales, plataformas tecnológicas de enseñanza-aprendizaje, herramientas computarizadas para la investigación y la innovación, redes electrónicas, etc.).

\section{CULTURAORGANIZACIONAL}

La cultura organizacional en la universidad está inmersa en un macro y supra sistema que permanentemente se expone a cambios sociales, económicos, tecnológicos e incluso políticos; realidad definida por valores, creencias, ideas, sentimientos y voluntades. La cultura posee tal poder porque puede determinar y condicionar el comportamiento de la universidad y definir la forma y esencia de pensar, sentir y actuar; de manera que la cultura incide, además, en la estructuración de la visión, las estrategias y las acciones. En síntesis, la cultura determina la forma y esencia de la universidad y, consecuentemente, la calidad de sus productos, procesos y servicios hacia adentro y hacia afuera; favoreciendo la conectividad e interacción con su entorno social, económico, político y ambiental; gestionando apropiadamente la diversidad y complejidad cultural en una relación lineal y en espiral de la coherencia, integración y cambio que propician y estimulan la competitividad. 


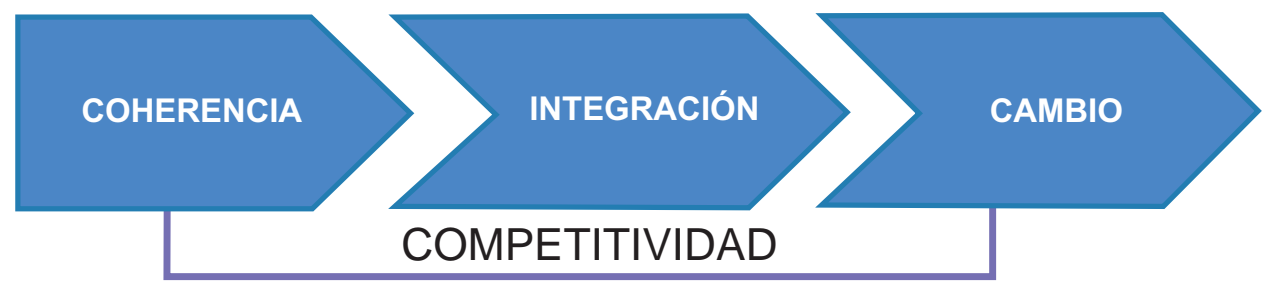

Para llevar a cabo el proceso de construcción de la cultura organizacional universitaria se debe estar al tanto de la filosofía, la ideología y las aspiraciones institucionales, y de cualquier síntoma que afecte cualquier intento de cambio.

A partir de aquí se deben diseñar y desarrollar las estrategias para dirigir el cambio.

\section{CAMBIO ORGANIZACIONAL}

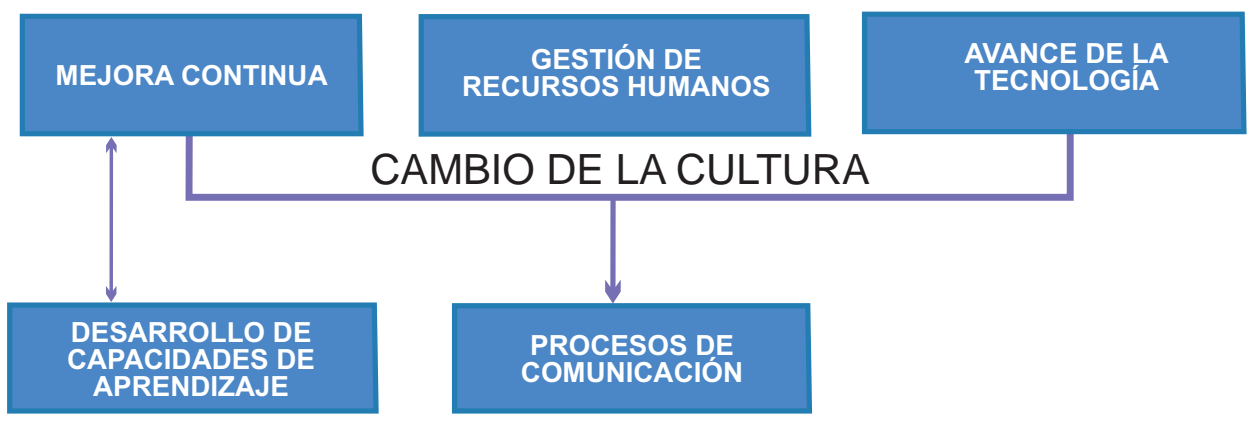

El cambio organizacional planteado es una estrategia que llevada a la práctica puede colocar a la universidad en el plano de la competitividad a partir de la generación de ideas innovadoras, validación, transferencia de conocimientos explícitos y tácitos al sector social y productivo para solucionar problemas y favorecer el desarrollo económico.

Desde luego que, para que la universidad alcance estándares que la vuelvan competitiva y eficaz, es necesario desarrollar las capacidades de gestión tecnológica y de proyectos; generar un sistema de exploración tecnológica de investigación y desarrollo, activar formas alternas de vinculación con el sector productivo, con el sector público y demás instituciones de educación superior. 


\section{CULTURA ORGANIZACIONAL}

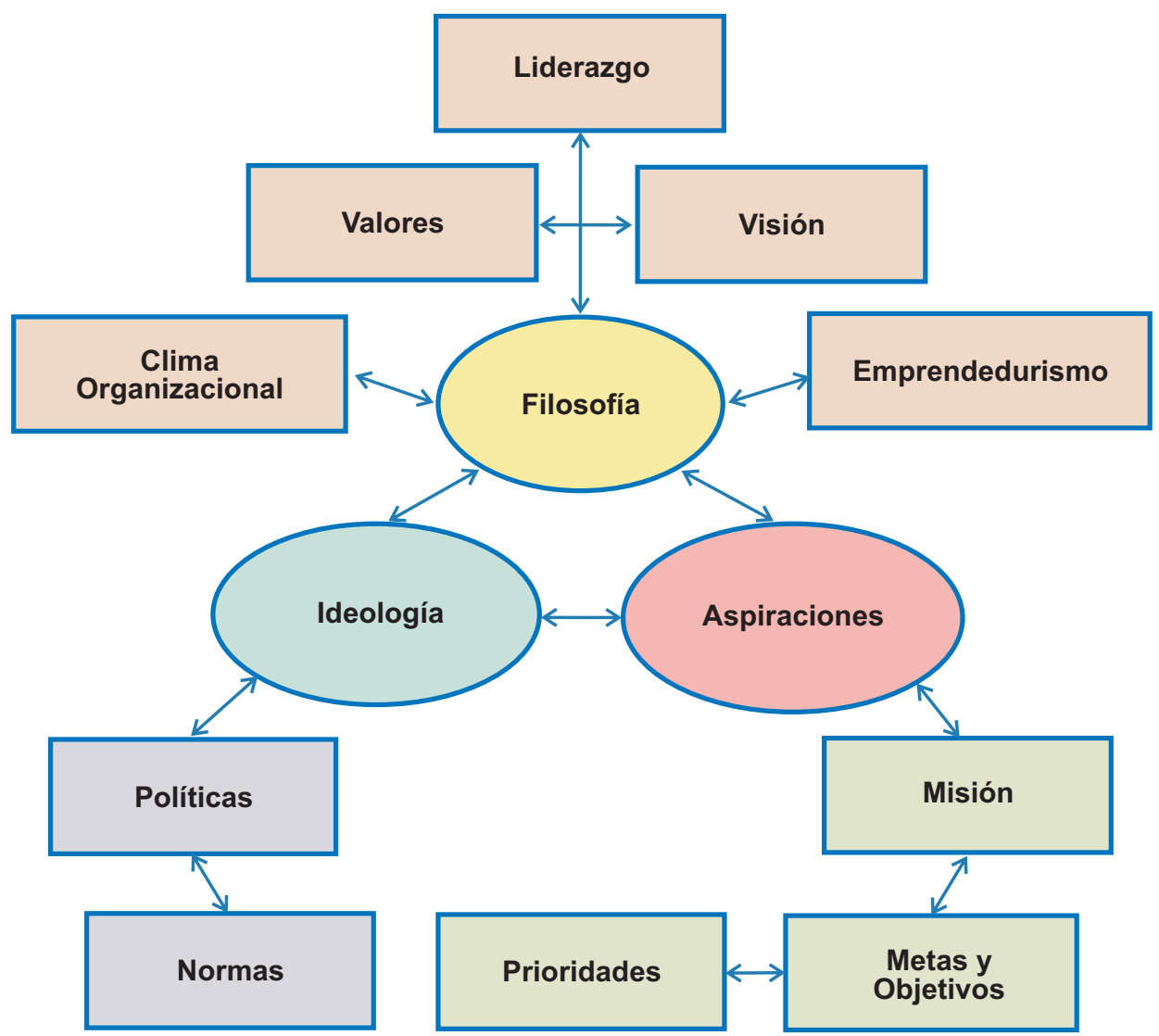

En la construcción de este enfoque organizacional se debe superar la tradición del cortoplacismo y, en la línea de tiempo, la visión debe plantearse en el mediano y largo plazo; en donde la gobernanza universitaria se aplane y se torne participativa. Ésto facilitará la ejecución de actividades orientadas a descubrir, capturar, compartir y aplicar conocimientos; poniendo de relieve la importancia de la explotación y protección del conocimiento científico y tecnológico cuya herramienta principal es la propiedad intelectual (Activo intangible valorado como el más importante de todos los que posee la institución o empresa pública o privada). Ha tomado tal importancia la propiedad intelectual que se aplica tanto para conceder licencias sobre productos y tecnologías, como para inhibir a terceros en su utilización.

La propiedad intelectual, además de su importancia económica, representa 
oportunidades en materia de acuerdos de cooperación en I+D, por lo que resulta importante fortalecer y desarrollar la cultura en Propiedad Intelectual sin perder de vista que estos activos intangibles se desarrollan en forma apropiada cuando se dispone de estrategias coherentes alineadas con las necesidades y demandas de la sociedad en su conjunto. El Sistema de Propiedad Intelectual es considerado como muy necesario para fomentar la creación intelectual en aras del interés público.

En este proceso las bases de datos sobre propiedad intelectual juegan un papel importante, ya que son fuente de información al servicio de la invención, la investigación, la enseñanza y el aprendizaje tanto de investigadores, docentes, estudiantes de pregrado, postgrado y agentes sociales y económicos demandantes de información especializada y calificada.

\section{EDUCACIÓNEINVESTIGACIÓN}

A pesar de los argumentos señalados, de nada serviría la formulación de una estrategia innovadora de la universidad si ésta continúa operando con sistemas rígidos de gestión, con áreas y funciones incomunicadas, llevando a cabo tareas disciplinarias en vez de interdisciplinarias, con una burocracia que privilegia trámites lineales de aprobación, desconociendo el sentido de la urgencia frente a lo importante, con esquemas ejecutivos tradicionales centrados en el consumismo de conocimientos; es decir la transmisión de conocimientos por medio de la docencia. Es urgente que quienes toman las decisiones en la universidad introyecten en sus constructos gerenciales que la innovación es el resultado de un proceso que combina elementos de la cultura, la organización, la investigación, el desarrollo y la transferencia de conocimientos. El desafío es empezar por incorporarlos al aprendizaje.

Parafraseando a Cortázar, J; (1988), "Cuando hablamos de educación no nos estamos refiriendo siempre a lo mismo, ni por su valor ni por el lugar desde donde se hable y/o argumente sobre la educación, pues no se trata del rescate de un pasado ideal como si se hubiese perdido una época de esplendor, sino más bien de dotar a la educación de una potencia diferente, de una dimensión nueva en la perspectiva de ubicarla como categoría que, irrumpiendo en el pensamiento, posibilite otras formas de pensar".

La universidad tiene planteado el gran reto de repensarse, de lo contrario estaría en una situación de agonía prolongada y eventualmente podría caer en coma, del cual difícilmente podría rescatarse; salvo por medio de sacrificios muy grandes por parte de la sociedad. Estamos conscientes que "predecir el futuro no es fácil, sin embargo 
pensar estratégicamente en lo que viene, ayuda a construir el futuro deseado". Entiendo que, "no hay verdades absolutas, sino muchas verdades sobre un mismo problema"; no obstante hay criterios que albergan sentidos epistemológicos que los aproxima con fuerza a la interpretación de la realidad histórica-concreta y a partir de allí se hacen planteamientos que vale la pena tomar en cuenta para la construcción de entramados estratégicos y la organización de procesos sostenibles.

Es incuestionable que la universidad, en la esencia de su quehacer, gira alrededor de: a) La formación, b) La investigación y c) La transferencia. Lo anterior, puede traducirse a lenguaje llano y decir que la formación equivale a la transferencia del conocimiento por medio de la educación y la tecnología; mientras que la investigación permite la producción de conocimiento científico y tecnológico, misión que estará incompleta si no agregamos la protección del conocimiento por medio de la propiedad intelectual y, desde luego, la innovación como expresión del valor agregado en el conocimiento.

\section{MAPACONCEPTUAL}

Sin el ánimo de plantear una receta rígida de cómo construir una Cultura de Investigación, desarrollo, innovación y propiedad intelectual, de manera sintética trazo un mapa conceptual que establece las bases fundamentales para el cambio que promueva la enseñanza y el aprendizaje de las ciencias y la tecnología de cara ala sociedad del conocimiento:

a) Diseño y desarrollo de un Currículo en coherencia con las tendencias del desarrollo social y económico; incluyendo nuevos contenidos, nuevos métodos de enseñanza y aprendizaje y nuevas formas de evaluación. Creación de programas de formación y capacitación de profesores, investigadores y gestores de procesos; fortalecimiento de la infraestructura de Ciencia y Tecnología, mejoramiento de los sistemas de información y comunicación (Las TIC), gestión de Cooperación por medio de proyectos de investigación, desarrollo tecnológico, modernizar los mecanismos de administración de recursos para la investigación científica, tecnológica e innovación, especial énfasis debe ponerse en diseño y ejecución de interfaces para la transferencia del conocimiento y la cooperación con actores de Ciencia, Tecnología; demandantes de productos, servicios y procesos para mejorar permanentemente la Competitividad del Sector productivo y problemas sociales y ambientales.

b) Proporcionar a los estudiantes información fidedigna y actualizada sobre 
diversas carreras y profesiones relacionadas con la Ciencia y la Tecnología (a nivel de pregrado incluir las interacciones entre Ciencia, Tecnología y Sociedad). Aplicar las técnicas de la Ciencia. En los Postgrados, la comprensión de la filosofía de la Ciencia y la Tecnología con sus conexos (La innovación y la propiedad intelectual en ambos niveles).

c) La dimensión cultural de la Ciencia, sus aplicaciones y las relaciones con la tecnología.

d) El estudio de la Ciencia en su contexto social, político y económico desde las demandas y necesidades de la sociedad.

e) La dimensión de las relaciones entre la educación científica, tecnológica y la sociedad.

f) Estimular el pensamiento crítico, la creatividad y la comunicación entre estudiantes y de éstos con los profesores.

g) Aspectos axiológicos y actitudes en la enseñanza de la Ciencia y la Tecnología; otorgando especial cuidado en dos elementos curriculares: Conocimientos sobre ciencia, Tecnología y actitudes éticas.

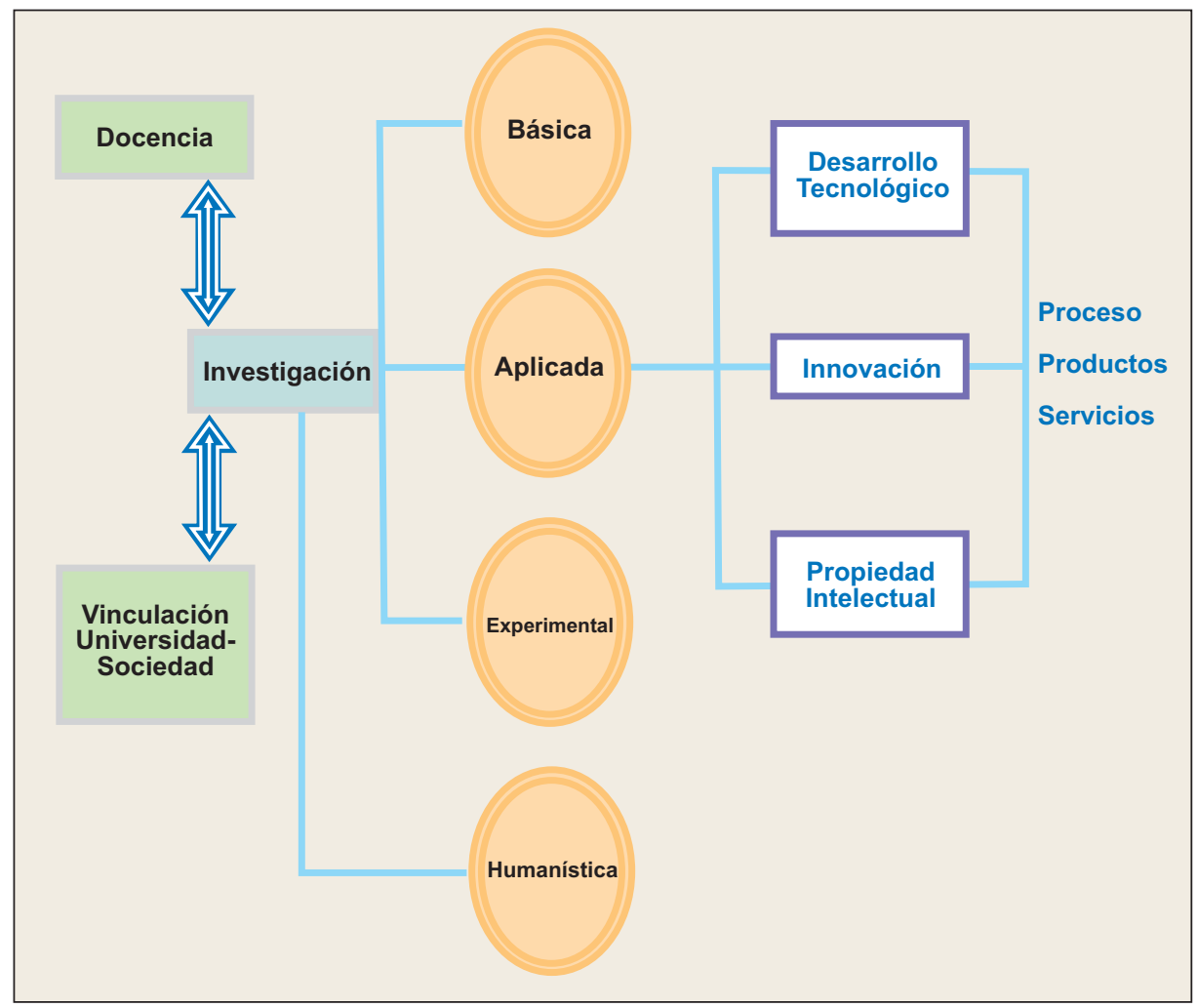




\section{CONCLUSIONES}

Esta arquitectura descansa y se apoya en la investigación, el desarrollo y la transferencia del conocimiento. Dichos elementos actúan sobre la cultura deinnovación y de la propiedad intelectual. Indudablemente, el planteamiento está centrado en el trabajo de la relación de contingencia, en donde se administran los intangibles: Capital Intelectual, el Aprendizaje de nuevas capacidades el Conocimiento y su relación con el entorno.

Cualquier análisis y decisión que gravite en el futuro de la universidad, debe someterse ineluctablemente al laboratorio crítico del pensamiento inteligente de todos los actores que componen la población universitaria y de sus aliados naturales y obligados (Sectores: social, productivo y público).

\section{BIBLIOGRAFÍA}

Acevedo, J.A. (1996). Cambiando la práctica docente en la enseñanza de las ciencias a través de CTS. Revista, 13, 26-30. En línea en Sala de Lecturas CTS+1 de la OEl, http://www.campus-oei.org/salactsi/acevedo2html, 2001

Caamaño, A. y Vilches, A. (2001). La alfabetización Científica y la Educación CTS:

Un elemento esencial de la cultura de nuestro tiempo. Enseñanza de las Ciencias, No. Extra, tomo 2 (VI Congreso).

Cortazar, J. (1988). Instrucciones para subir una escalera, en historias de cronopios y de famas, Madrid, Alfaguara.

Martinez, B. Alberto.(2001). De la escuela expansiva a la escuela competitiva en América Latina. Universidad Pedagógica Nacional, Bogotá.

Habermas, Jurgen. (1989). El discurso filosófico de la modernidad, Buenos Aires, Taurus.

Universidad de Campinas. (2009). Gestión de Propiedad Intelectual en Instituciones de Educación Superior, Buenas Prácticas en Universidades de Latinoamérica y Europa. http//www.unicamp.br/unicamp/.

Red de Propiedad Intelectual e Industrial en Latinoamerica, (PILA). (2009). Materiales de Formación. Fundamentos de la PI. 